\title{
Electron Channeling Contrast Imaging for Non-Destructive Analysis of Extended Defects in Semiconductor Thin Films and Device Structures
}

\author{
M.B. Katz ${ }^{1}$, M.E. Twigg ${ }^{1}$, S.I. Maximenko ${ }^{1}$, N.D. Bassim² ${ }^{2}$, N.A. Mahadik ${ }^{1}$, G.G. Jernigan ${ }^{1}$, C.L. \\ Canedy $^{3}$, J. Abell $^{3}$, and C.A. Affouda ${ }^{1}$ \\ ${ }^{1}$ Electronics Science \& Technology Division, United States Naval Research Laboratory, Washington, \\ DC 20010 \\ 2.Materials Science \& Technology Division, United States Naval Research Laboratory, Washington, DC \\ 20010 \\ ${ }^{3}$ Optical Sciences Division, United States Naval Research Laboratory, Washington, DC 20010
}

Electron Channeling Contrast Imaging (ECCI) [1] offers the ability to perform fast and non-destructive analysis of the type and density of extended defects, such as threading dislocations (TDs) and stacking faults (SFs) penetrating as-grown semiconductor thin film surfaces and device structures. Using a conventional scanning electron microscope (SEM) chamber with only a few special requirements, ECCI utilizes a glancing-angle geometry and diode electron detectors placed in the forescatter position to collect Bragg-diffracted electrons. Contrast arises when crystal defects, such as TDs and surface steps, perturb the nominal crystal structure, with the resulting diffraction-contrast effects manifested as bright or dark features in the resulting image [2]. Furthermore, the type of TD or SF can be deduced from the specific contrast patterns in this image, and comparison with simulation [3].

Because ECCI-compatible sample mounts can orient pieces of semiconductor wafers as large as a few centimeters in diameter in most machines, and that these samples require no preparation, the technique can be used to monitor device fabrication and screen out possible problems. Maps of extended defects produced by EECI can also be compared with data acquired via Electron Beam Induced Current (EBIC), Transmission Electron Microscopy (TEM), and X-ray Diffraction (XRD). In addition, ECCI is able to operate at relatively low magnifications, allowing large areas to be quickly examined for defects occurring at low densities. These qualities, taken together, make ECCI an excellent technique with which to study defects in semiconductor films and devices.

Our study focuses on ECCI, TEM, XRD, and EBIC analyses of films grown by molecular beam epitaxy. SiGe layers deposited on (100) Si substrates are seen to generate threading dislocations (TDs) that penetrate the sample surface at angles corresponding to $<110>$ directions. For TDs in InGaSb graded layers grown on (100) GaSb substrates, however, the TDs are found to be perpendicular to the sample surface.

[1] M.A. Crimp, Microscopy Research and Techniques 69 (2006) 374

[2] Y.N. Picard, et al., Appl. Phys. Lett. 90 (2007) 234101

[3] M.E. Twigg and Y.N. Picard, J. Appl. Phys. 105 (2009) 093520

[4] The authors would like to thank the NRL Institute for Nanoscience for maintenance of the SEM instrument. MBK would like to thank J.K. Hite at NRL for assistance with ECCI. 

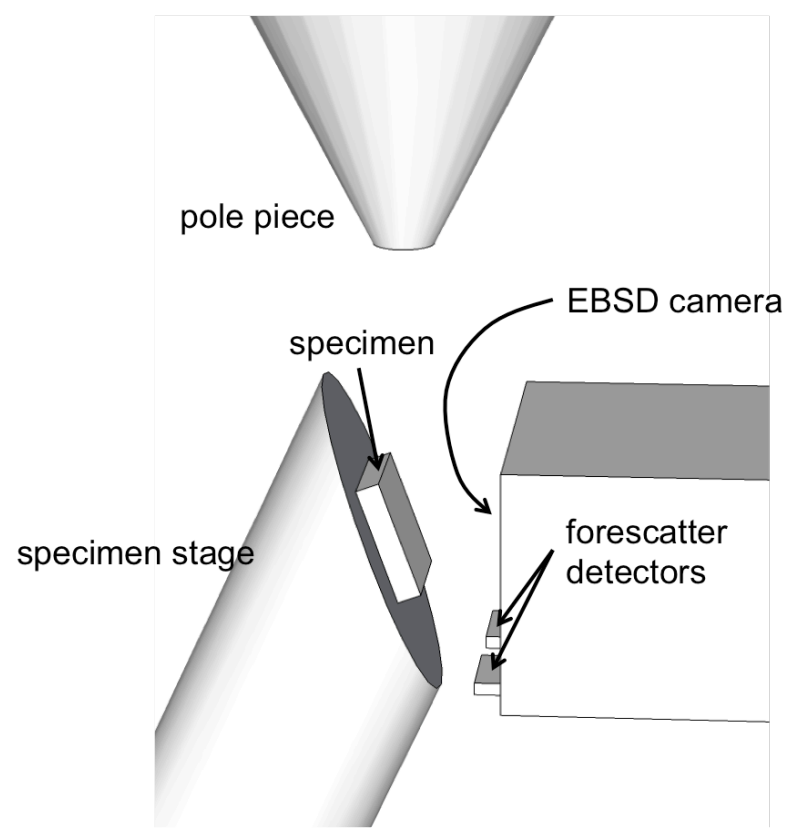

Figure 1. Schematic of the ECCI forescatter geometry.

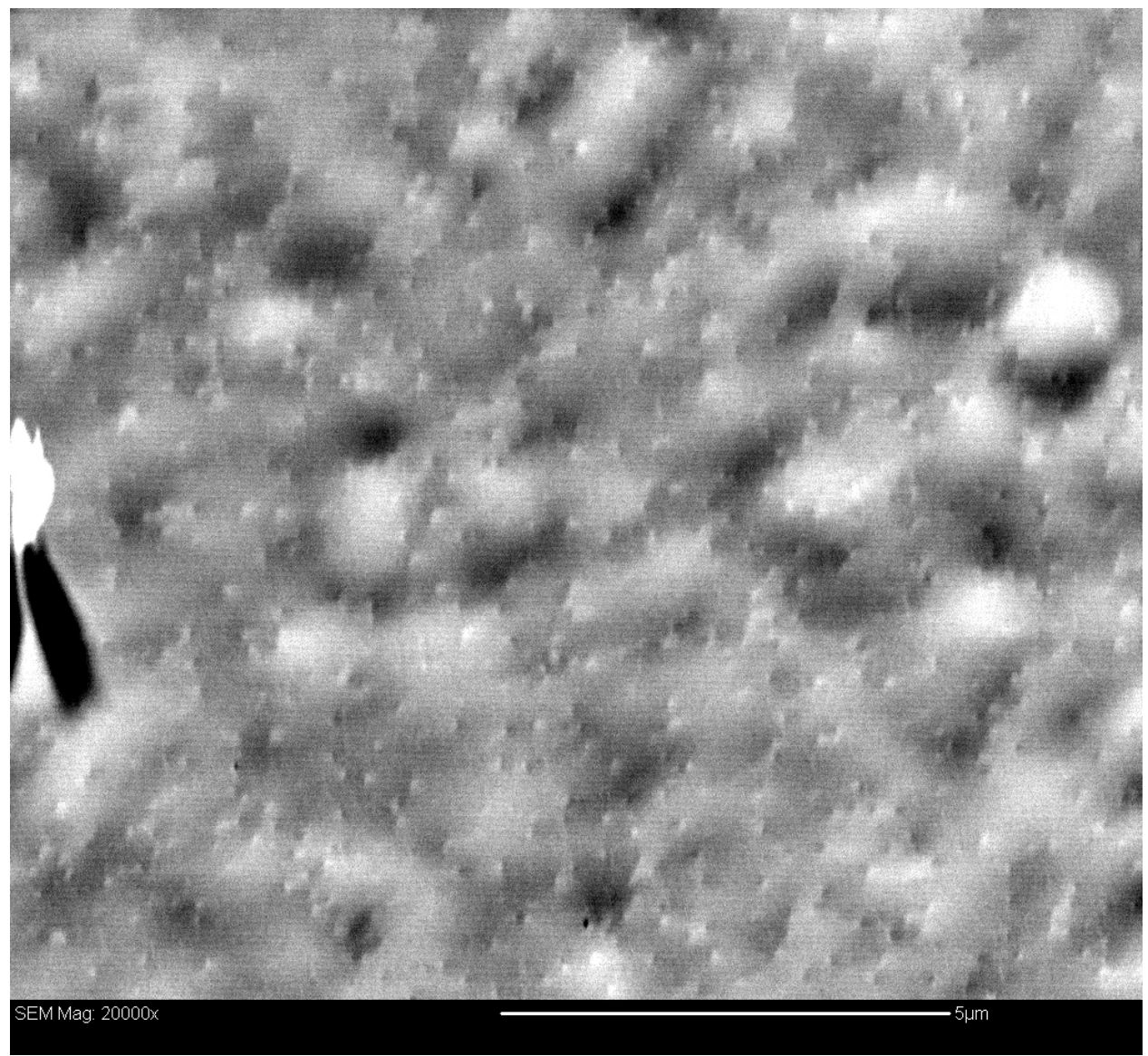

Figure 2. ECCI micrograph of a Ge film on Si. Threading dislocations manifest as bright dots in the image. (The bright feature on the left is surface debris and the adjacent dark contrast is an electron shadow it creates.) 